ARTICLE

\title{
DELLA-mediated PIF degradation contributes to coordination of light and gibberellin signalling in Arabidopsis
}

Kunlun $\mathrm{Li}^{1}$, Renbo $\mathrm{Yu}^{1}$, Liu-Min Fan ${ }^{1}$, Ning Wei ${ }^{2}$, Haodong Chen ${ }^{1} \&$ Xing Wang Deng ${ }^{1}$

Light and gibberellins (GAs) antagonistically regulate hypocotyl elongation in plants. It has been demonstrated that DELLAs, which are negative regulators of GA signalling, inhibit phytochrome-interacting factors 3 and 4 (PIF3 and PIF4) by sequestering their DNA-recognition domains. However, it is unclear whether there are other mechanisms of regulatory crosstalk between DELLAs and PIFs. Here, we demonstrate that DELLAs negatively regulate the abundance of four PIF proteins through the ubiquitin-proteasome system. Reduction of PIF3 protein abundance by DELLAs correlates closely with reduced hypocotyl elongation. Both sequestration and degradation of PIF3 by DELLAs contribute to a reduction in PIF3 binding to its target genes. Thus, we show that promotion of PIF degradation by DELLAs is required to coordinate light and GA signals, and the dual regulation of transcription factors by DELLAs by both sequestration and degradation may be a general mechanism.

\footnotetext{
${ }^{1}$ State Key Laboratory of Protein and Plant Gene Research, Peking-Tsinghua Center for Life Sciences, School of Advanced Agriculture Sciences, and School of Life Sciences, Peking University, Beijing 100871, China. ${ }^{2}$ Department of Molecular, Cellular and Developmental Biology, Yale University, New Haven, Connecticut 06520, USA. Correspondence and requests for materials should be addressed to H.C. (email: chenhaodong@pku.edu.cn) or to X.W.D. (email: deng@pku.edu.cn).
} 
ight promotes plant photomorphogenesis, giving rise to open and expanded cotyledons, and short hypocotyls in light-grown Arabidopsis seedlings. In the dark, seedlings undergo skotomorphogenesis/etiolation, as characterized by closed cotyledons and elongated hypocotyls ${ }^{1}$. A subset of basic helix-loop-helix (bHLH) transcription factors, known as phytochrome-interacting factors (PIFs), has been reported to have a key role in etiolation and light-regulated plant development. PIF3 was the first characterized member of this gene family, identified by yeast two-hybrid screen using phytochrome $\mathrm{B}$ as the bait ${ }^{2}$. Mutation of PIF3 resulted in short hypocotyls in red light, indicating that PIF3 is a negative regulator of light signal transduction ${ }^{3,4}$. Several other homologous PIF proteins, including PIF1, PIF4, and PIF5, have also been reported to regulate photomorphogenesis ${ }^{5-7}$. A quadruple pif mutant (lacking PIF1, PIF3, PIF4, and PIF5) exhibits a striking constitutively photomorphogenic phenotype in the dark, which indicates that these four PIF factors act redundantly to promote etiolated growth ${ }^{8-10}$. In the dark, PIF proteins accumulate and directly regulate thousands of genes to maintain skotomorphogenesis $^{11-13}$. Upon illumination, the photoactived phytochromes trigger PIFs' rapid phosphorylation and subsequently proteasome-mediated degradation ${ }^{14-17}$, leading to a cascade of transcriptional changes to promote photomorphogenesis ${ }^{12,18-20}$.

During seedling development, gibberellins (GAs) promote etiolated growth, thus showing the opposite effect to light on photomorphogenic programme ${ }^{21}$. Deficiency of GA (as in ga1-3 mutant) induces partial constitutive photomorphogenesis phenotype in darkness, resulting in a loss of apical hook, open cotyledons and shortened hypocotyls ${ }^{21}$. DELLA proteins are the key repressors of almost all GA responses ${ }^{22}$. There are five DELLA proteins in Arabidopsis GA INSENSITIVE (GAI), REPRESSOR OF ga1-3 (RGA), RGA-LIKE 1 (RGL1), RGL2 and RGL3, which have both distinct and overlapping functions ${ }^{22-}$ 30. In darkness, constitutively photomorphogenic phenotypes of ga1-3 mutants can be almost fully suppressed by rga and gai null alleles, indicating that RGA and GAI are the two main DELLA members involved in GA-dependent repression of photomorphogenic growth in seedlings ${ }^{21,31,32}$. When GA is present, GA receptor GID1 binds to the DELLAs to form GID1GA-DELLA complex, which triggers the ubiquitination and subsequent degradation of DELLA proteins by the $26 \mathrm{~S}$ proteasome $\mathrm{s}^{33-35}$. DELLAs have a conserved DELLA domain at the $\mathrm{N}$ terminus that is essential for GA-triggered protein degradation $^{22}$. Deletion of the DELLA domain results in stabilization of these proteins and leads to a GA-unresponsive dwarf phenotype ${ }^{22}$.

How light and GAs antagonistically regulate hypocotyl elongation has been extensively analyzed in the past two decades. Two independent studies demonstrated that DELLAs can physically interact with and block PIF3 and PIF4 activities by sequestering the transcription factors from binding to their targets, which ultimately results in inhibition of hypocotyl elongation ${ }^{36,37}$. Further study showed that DELLAs also interact with the bHLH proteins PIF1 (also known as PIL5), PIF6 (also known as PIL2) and SPATULA $(\mathrm{SPT})^{38}$. The sequestration of PIFs by DELLAs provided an important molecular link between light and GA signalling in regulating photomorphogenesis. Later, the model of sequestration was demonstrated to be a general mechanism for DELLAs to regulate transcription factors involved in other signalling pathways ${ }^{39-44}$. Beside the well-established sequestration action model, we are interested in exploring whether there are additional regulatory actions between DELLAs and PIFs in the crosstalk of light and GA signals.

We were intrigued by the observation that RGA and GAI proteins oscillate in a diurnal manner, with higher level during daytime and lower level at night, which is critical for the rhythmic growth of hypocotyls ${ }^{45}$. In contrast, as the prominent regulators of seedlings growth under the diurnal condition, PIF3 abundance accumulates in the dark to induce hypocotyls growth and it decreases after light illumination ${ }^{46}$. Following the clue of opposite oscillation patterns of DELLAs and PIF3 proteins, we focused on protein turnover as a potential regulatory mechanism. In this study, we report that DELLAs promote the degradation of PIF proteins through the ubiquitin-proteasome system. Thus, protein degradation is an additional mechanism by which DELLAs inactivate PIFs to coordinate light and GA signals during plant development.

\section{Results}

DELLAs negatively regulate PIF3 protein abundance. Intrigued by opposite protein accumulation dynamics of DELLAs and PIF3 in a diurnal cycle ${ }^{45,46}$, we designed the experiments to ask whether DELLAs and PIF3 affect the protein level of each other. Two DELLA proteins RGA and GAI are the two main repressors of GA-mediated suppression of photomorphogenesis in darkness $^{21}$, were used for the study. The GA-insensitive mutants $r g a-\Delta 17$ and gai, lacking 17 amino acids within the DELLA domain that is required for GA-induced degradation $23,24,26$, were used to examine PIF3 protein level. As shown before ${ }^{26}$, the rga- $\Delta 17$ mutant exhibited shorter hypocotyls compared to its wild-type counterpart, Ler in darkness (Fig. 1a). Accompanying this phenotype, endogenous PIF3 protein levels were dramatically reduced (Fig. 1b). Similarly, the non-degradable GAI mutant showed shortened hypocotyls and decreased PIF3 protein abundance in darkness (Fig. 1a,c). On the basis of the observed effects on hypocotyl length and PIF3 protein abundance, it seems that RGA has a more dominant role than GAI in inhibiting skotomorphogenesis. By contrast, della pentuple mutant exhibited elongated hypocotyl length and slightly increased PIF3 protein abundance compared with the wild type (Fig. 1a,b), further supporting a negative role of DELLAs in regulating skotomorphogenesis and PIF3. RGA and GAI had little effect on PIF3 transcript level (Supplementary Fig. 1a,b), suggesting that DELLAs negatively regulate PIF3 abundance mainly at the post-transcriptional level. To test whether PIF3 affects the abundance of RGA, we examined RGA protein levels in wild type, 35:PIF3-Myc, pif3-3 and pifq (pif1 pif3 pif4 pif5) seedlings, and the results suggested that PIF3 has no obvious effect on RGA protein level (Fig. 1d).

Induction of DELLA proteins triggers PIF3 degradation. To further investigate the role of DELLAs in regulating PIF3 protein abundance, we generated transgenic Arabidopsis plants harbouring the HA-tagged RGA $\Delta 17$ or GAI $\Delta 17$ under the control of a dexamethasone (DEX)-inducible promoter. RGA $\Delta 17-\mathrm{HA}$ and GAI $\Delta 17-\mathrm{HA}$ seedlings without treatment showed typical etiolated phenotypes in darkness, while DEX-treated seedlings displayed retarded hypocotyl growth (Fig. 2a,b), similar to rga- $\Delta 17$ or gai mutants (Fig. 1a). Immunoblot analysis showed that both RGA $\Delta 17-\mathrm{HA}$ and GAI $\Delta 17-\mathrm{HA}$ proteins accumulated to high levels after DEX induction (Fig. 2c,d). These results demonstrated that DEX-induced RGA $\Delta 17-\mathrm{HA}$ and GAI $\Delta 17-\mathrm{HA}$ fusion proteins were biologically functional, as they were able to shortcut endogenous GA action and induce a photomorphogenic-like phenotype. The amount of PIF3 was markedly decreased when RGA $\Delta 17-H A$ or GAI $\Delta 17$ HA accumulated in the presence of DEX (Fig. 2c,d), which is consistent with the results in Fig. 1. We next performed transient induction of the transgenes. After 12 or $24 \mathrm{~h}$ DEX treatment, as RGA $\Delta 17-H A$ protein accumulated, PIF3 protein abundance 
a
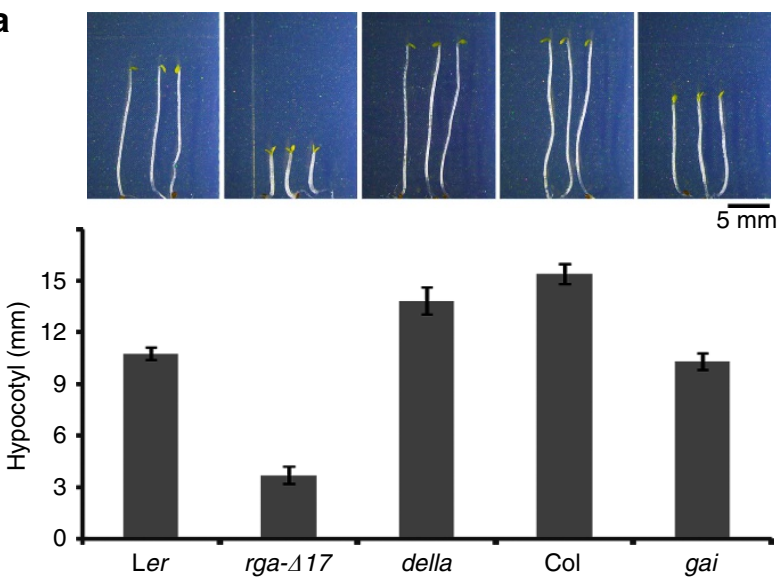

b

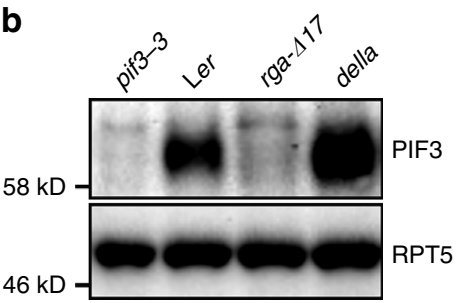

C

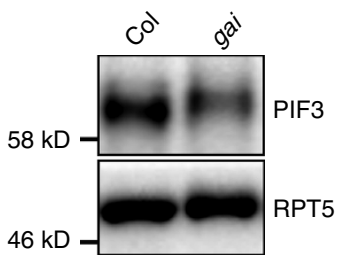

d

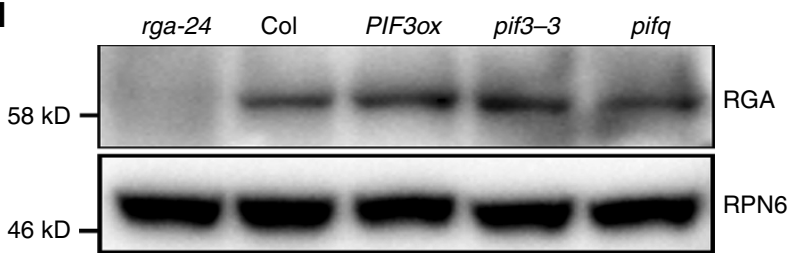

Figure 1 | DELLAs negatively regulate PIF3 protein abundance in the dark. (a) Phenotypes and hypocotyl lengths of 4-day-old dark-grown rga- $\Delta 17$, della, gai and their wild-type counterparts. Means \pm s.d. were obtained from at least 30 seedlings. (b,c) Endogenous PIF3 protein levels in various DELLA mutants. Total proteins extracted from 4-day-old darkgrown seedlings were immunoblotted with anti-PIF3 and anti-RPT5 antibodies. RPT5 was used as a loading control. (d) Effects of PIFs on RGA protein levels. Four-day-old dark-grown seedlings were collected for protein extraction, and total proteins were immunoblotted with anti-RGA and anti-RPN6 antibodies. PIF3ox indicates 35S:PIF3-Myc. RPN6 was used as a loading control. Each immunoblot result is the representative of at least three repeats.

dramatically reduced (Fig. 2e). Similarly, the accumulation of GAI $\Delta 17-H A$ after $24 \mathrm{~h}$ DEX treatment also led to the reduction of PIF3 protein (Fig. 2f). There were no obvious changes of PIF3 transcripts during the DEX induction (Supplementary Fig. 1c,d), indicating that DELLAs negatively regulated PIF3 mainly at the protein level.

DELLA control of PIF levels correlates with hypocotyl length. As the central repressors in the GA signalling pathway, abundance of DELLAs is tightly controlled by $\mathrm{GA}^{33-35}$. Since DELLAs were shown to regulate PIF3 protein abundance (Figs 1 and 2) and PIF3 promotes hypocotyls elongation ${ }^{3,46}$, we speculated that the abundance of PIFs might be regulated by GA content, which may further mediate hypocotyl elongation. As expected, $\mathrm{GA}_{3}$ (an active form of GA) treatment promoted, whereas paclobutrazol (PAC; a GA biosynthesis inhibitor) treatment inhibited hypocotyl elongation of wild-type (Col) plants (Fig. 3a and Supplementary Table 1). As shown in Fig. $3 b, \mathrm{GA}_{3}$ treatment promoted endogenous PIF3 protein accumulation, while PAC treatment caused an obvious decrease of PIF3 abundance. Consistently, GA 3 or PAC treatment of seedlings of two other wild-type Arabidopsis ecotypes, Wassilewskija (Ws) and Landsberg erecta (Ler), showed similar results (Supplementary Fig. 2). To exclude the possibility of regulation at the transcript level, 35S:PIF3-Myc seedlings were also treated with $\mathrm{GA}_{3}$ or PAC. Similar to the results in wild type (Fig. 3b), GA $A_{3}$ increased, while PAC repressed, PIF3-Myc protein abundance (Fig. 3c).

To quantitatively assess the relationships between DELLAs, PIF3 and hypocotyl elongation, we examined RGA and PIF3 protein levels, and hypocotyl length of 35S:PIF3-Myc seedlings grown in the presence of various PAC concentrations. With increasing dose of PAC, hypocotyl elongation of dark-grown seedlings declined progressively (Fig. $3 \mathrm{~d}$ ). In these seedlings, RGA accumulated, while PIF3-Myc decreased gradually (Fig. 3e). By calculating the relative change of hypocotyl length and protein abundance of RGA and PIF3-Myc, we found that the attenuating effect of RGA on PIF3 abundance correlated well with hypocotyl growth (Fig. 3f).

To further analyze the significance of DELLA's negative regulation on PIF3 abundance in the GA signalling pathway, we checked the phenotypes and PIF3 protein abundance in darkgrown sly1-10, sly1-10 gai-t6, sly1-10 rga-24 and sly1-10 gai-t6 rga-24 seedlings (Fig. 3g,h). SLEEPY1 (SLY1) is an E3 ligase component responsible for the ubiquitination of DELLA proteins, and its mutant (sly1-10) contains elevated levels of RGA and $\mathrm{GAI}^{33-35}$. The constitutive photomorphogenic phenotype of sly1-10 could be partially suppressed by the absence of GAI (gai-t6) and RGA (rga-24) (Fig. 3g), which indicates that accumulation of GAI and RGA can account for the short hypocotyl phenotype of sly1-10. PIF3 protein abundance was then examined in these mutants and the extremely low level of PIF3 in sly1-10 was partially rescued by the absence of RGA and GAI (Fig. 3h). This is consistent with the notion that over-accumulation of RGA and GAI proteins in sly1-10 promote degradation of PIF3. For the regulation of PIF3 protein level and hypocotyl elongation, the effect of RGA is stronger than GAI (Fig. 3g,h), similar to the results in Figs 1 and 2. These data suggest that the reduction of PIF3 abundance by DELLAs may have a key role in suppressing GA-mediated hypocotyl elongation.

DEX-induced RGA accumulation led to the PIF3 degradation within $12 \mathrm{~h}$ (Fig. 2). To determine how fast DELLAs' regulation of PIF3 responds to the change of GA levels, we applied $\mathrm{GA}_{3}$ to seedlings grown on medium containing PAC and checked how PIF3 level recovered. In the plants with PAC treatment, RGA protein accumulated and PIF3 protein decreased. $\mathrm{GA}_{3}$ application led to a quick reduction of RGA protein levels, while PIF3 abundance increased within hours and almost fully recovered by $12 \mathrm{~h}$ of $\mathrm{GA}_{3}$ treatment (Fig. 3i). Together, the data in Figs 2 and 3 suggest that DELLA-mediated degradation may provide the plant with a tunable way of regulating the abundance of PIFs in response to a variety of cues that modulate GA metabolism.

DELLAs contribute to diurnal oscillation of PIF3 levels. To investigate whether DELLAs also promote PIF3 degradation under light conditions, 35S:PIF3-Myc seedlings were grown on medium containing $\mathrm{GA}_{3}$ or PAC under continuous red light and PIF3-Myc protein levels were checked. As shown in Fig. 4a,b, DELLAs negatively regulate hypocotyl elongation and PIF3 protein abundance under red light. Considering that DELLAs and PIF3 show opposite protein abundance dynamics in a diurnal cycle $^{45,46}$, we were interested in whether DELLAs help forming the oscillatory wave of PIF3 levels under diurnal conditions. To 
a

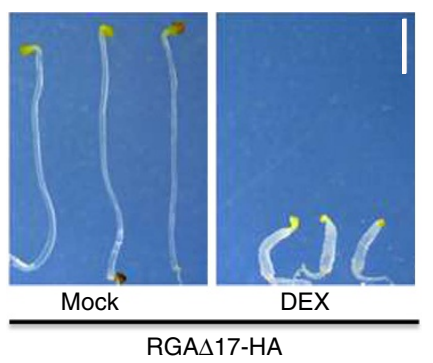

b

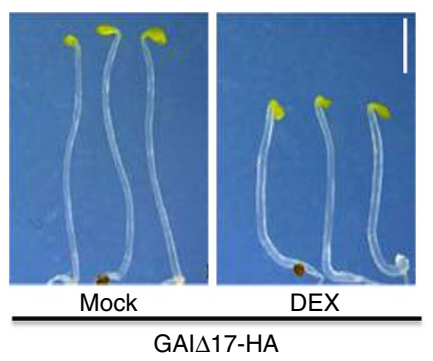

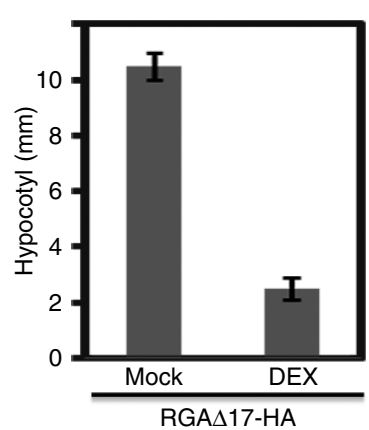

RGA $\Delta 17-H A$

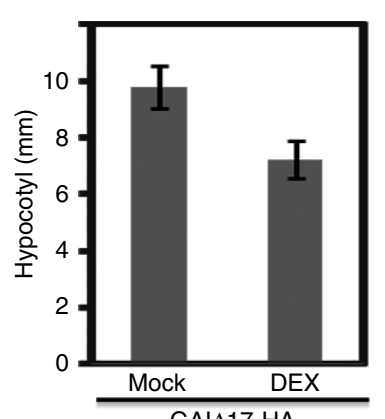

C

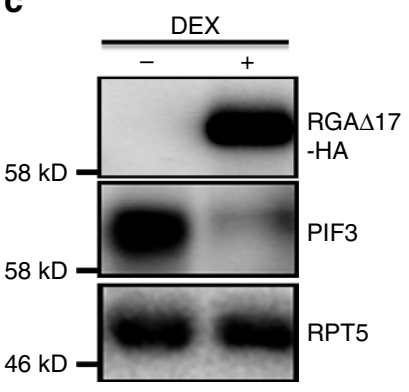

d

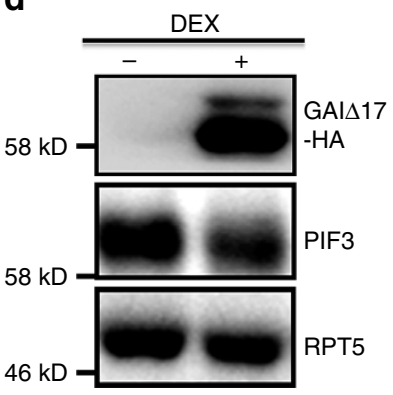

e

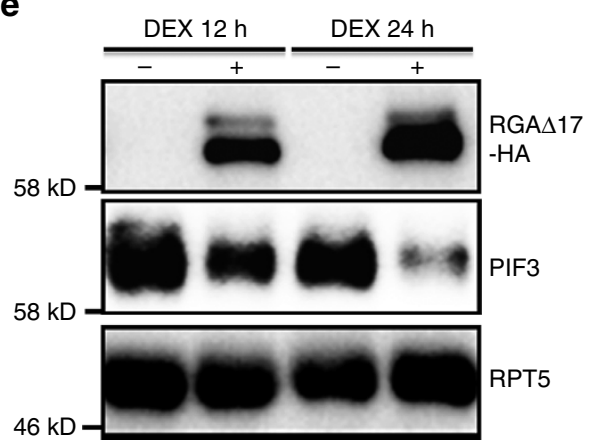

f

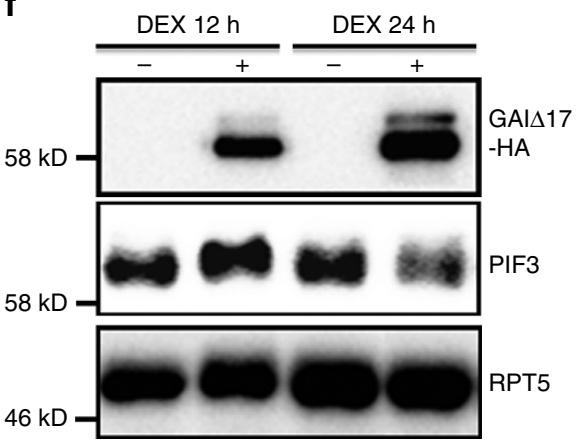

Figure 2 | Induction of DELLAs expression leads to decline of PIF3 protein levels in the dark. (a,b) Phenotypes and hypocotyl lengths of 4-day-old RGA $\Delta 17-\mathrm{HA}$ and GAI $\Delta 17-\mathrm{HA}$ seedlings treated without or with $1 \mu \mathrm{M}$ DEX in the dark. Means $\pm \mathrm{s}$.d. were calculated from at least 30 seedlings. All images were under the same magnification. Scale bar, $2 \mathrm{~mm}$. (c,d) RGA $\Delta 17-\mathrm{HA}, \mathrm{GAI} \Delta 17-\mathrm{HA}$ and PIF3 protein levels in the plants as shown in (a) and (b). Total proteins were analyzed by immunoblots using anti-HA, anti-PIF3 and anti-RPT5. (e,f) Analysis of PIF3 protein levels after transient induction of RGA $\triangle 17-\mathrm{HA}$ or GAI $\Delta 17-\mathrm{HA}$. Four-day-old dark-grown RGA $\Delta 17-\mathrm{HA}$ or GAI $\Delta 17-\mathrm{HA}$ seedlings were collected and infiltrated without or with $10 \mu \mathrm{M}$ DEX for $12 \mathrm{~h}$ or $24 \mathrm{~h}$. Total proteins were analyzed by immunoblots using anti-HA, anti-PIF3 and anti-RPT5. Each immunoblot result is the representative of at least three repeats, and RPT5 was used as a loading control.

test this, we examined the pattern of PIF3-Myc protein levels in 35S:PIF3-Myc seedlings treated with or without $\mathrm{GA}_{3}$ or PAC under diurnal short-day conditions. As shown in Fig. 4c,d, PIF3-Myc protein abundance was low in daytime and accumulated to higher levels in the night, which was consistent with previous studies ${ }^{46}$. On $\mathrm{GA}_{3}$ application, PIF3-Myc increased throughout the day compared to mock treatment, and the increase was larger in daytime and early night (at ZT4, 8, 12 h) than in mid- and late night (at ZT18, 24 h) (Fig. 4c,d), which weakened the oscillation patterns of PIF3 protein. These results suggested that DELLAs contribute to the oscillation patterns of PIF3 protein levels in plants grown in diurnal light/dark conditions. On PAC treatment, a condition that increased DELLA abundance, not only did PIF3-Myc levels decrease sharply, but also its oscillation pattern was dramatically impaired (Fig. 4c,d). These results further support the contribution of DELLAs to PIF3 oscillation under diurnal conditions.
DELLAs and phyB/LRBs mediate PIF degradation independently. We next tested whether DELLAs modulate the abundance of PIF3 via the ubiquitin-proteasome system. We found that PACinduced reduction of endogenous PIF3 levels in wild-type seedlings or seedlings ectopically expressing the PIF3-Myc protein was inhibited by treatment with MG132, a $26 \mathrm{~S}$ proteasome inhibitor (Fig. 5a,b). Similarly, MG132 treatment also increased PIF3 protein levels in rga- $\Delta 17$ mutant seedlings in the dark (Fig. 5c). Moreover, MG132 completely blocked PIF3 degradation in RGA $\triangle 17$-HA seedlings with DEX treatment (Fig. 5d). Taken together, these results showed that inhibiting proteasome activity is able to abolish the degradation of PIF3, which would otherwise take place under circumstances where DELLA proteins accumulate, and thus suggests that DELLAs promote PIF3 protein degradation through the ubiquitin-proteasome pathway.

Since PIF3 functions redundantly with its three homologues, PIF1, PIF4 and PIF5, to repress photomorphogenesis in darkness $^{8-10}$, and DELLAs sequester and inhibit transcriptional 
a

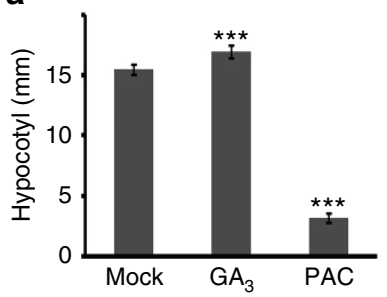

b

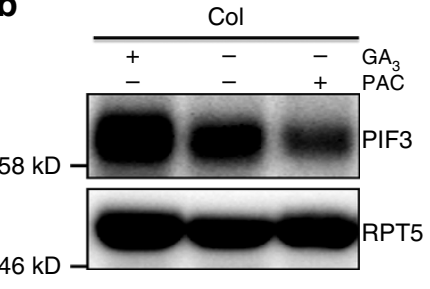

c

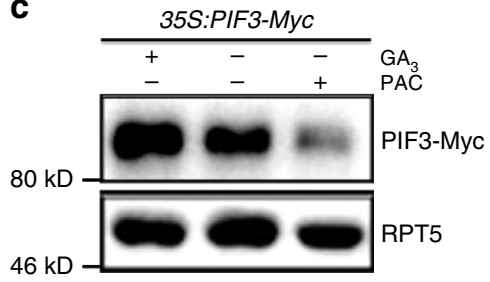

d
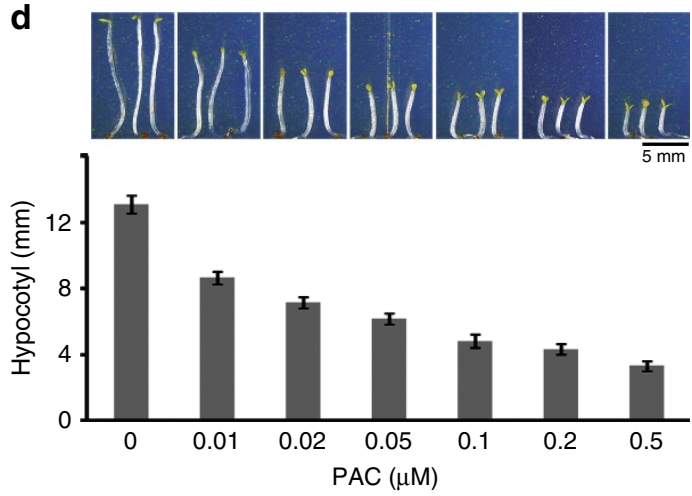

g
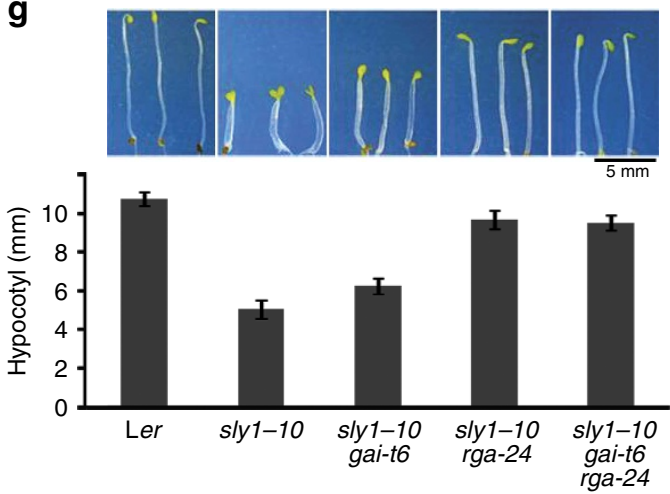

h

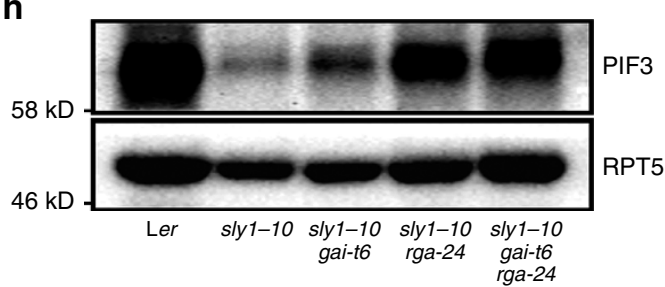

e

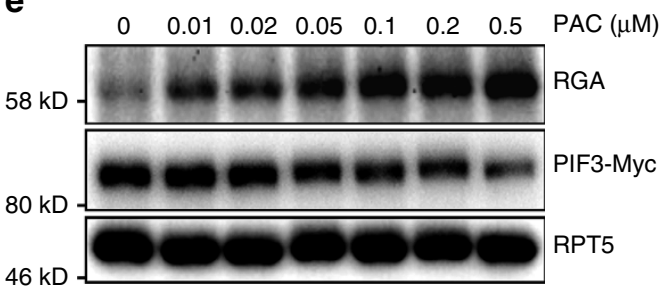

$\mathbf{f}$

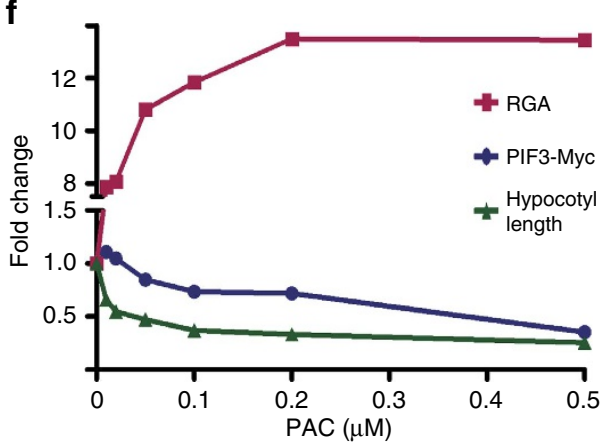

i

$58 \mathrm{kD}$

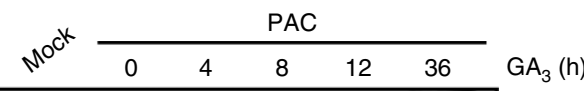

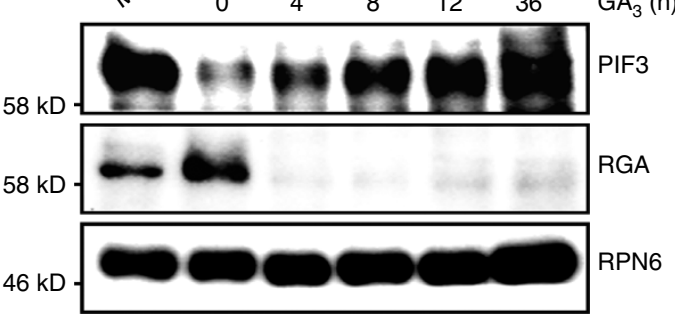

Figure 3 | Regulation of PIF3 protein abundance by DELLAs correlates with GA-regulated hypocotyl elongation. (a) Hypocotyl lengths of 4-day-old dark-grown wild-type $(\mathrm{Col})$ seedlings treated with $\mathrm{GA}_{3}(10 \mu \mathrm{M})$ or PAC $(0.5 \mu \mathrm{M})$. Statistical significance was determined using the Student's $t$ tests between the $\mathrm{GA}_{3}$ or PAC treatment and Mock. ${ }^{\star * \star} \mathrm{P}<0.001$. (b,c) Effects of $\mathrm{GA}_{3}$ or PAC treatment on PIF3 and PIF3-Myc protein abundance. The seedlings were grown in the medium with indicated supplements, and total proteins were analyzed by immunoblots using anti-PIF3, anti-Myc and anti-RPT5. (d) Phenotypes and hypocotyl lengths of 4-day-old dark-grown 35S:PIF3-Myc seedlings treated with different concentrations (0, 0.01, 0.02, $0.05,0.1,0.2$ and $0.5 \mu \mathrm{M}$ ) of PAC. All images were under the same magnification. (e) RGA and PIF3-Myc protein levels of the seedlings as shown in (d). (f) Relative changes of RGA, PIF3-Myc, and hypocotyl lengths of the seedlings shown in (d,e). The values of starting points were set to 1. (g) Phenotypes and hypocotyl lengths of 4-day-old dark-grown seedlings. All images were taken under the same magnification. (h) Endogenous PIF3 protein levels of the seedlings shown in (g). (i) PIF3 protein levels in Col seedlings treated with PAC and GA. Seedlings were grown on $0.2 \mu \mathrm{M}$ PAC-containing medium in darkness for 4 days and then treated with $100 \mu \mathrm{M} \mathrm{GA}$ for indicated times. Each immunoblot result is the representative of at least three repeats, and RPN6 was used as a loading control. In (a), (d) and (g), Means \pm s.d. were obtained from at least 20 seedlings.

activity of both PIF3 and PIF4 (refs 36,37), we tested whether DELLAs also negatively regulate protein abundance of PIF1, PIF4 and PIF5. As shown in Fig. 5e-g, the abundance of all three PIFMyc proteins was reduced in the 35S:PIF1-Myc, 35S:PIF4-Myc and 35S:PIF5-Myc seedlings after PAC treatment. Furthermore, PAC triggered reduction of PIF-Myc abundance could be inhibited by MG132 treatment. Collectively, these data indicate that DELLAs promote the degradation of these four PIF proteins via the proteasome.

The light-activated photoreceptor phyB interacts with PIF3 and recruits LRB1/2/3 containing E3 ligases to ubiquitinate PIF3 (ref. 16). To address whether phyB and LRBs are involved in DELLA-mediated PIF degradation, we treated phyB-9 and $l r b 123$ mutants with different concentrations of PAC (Fig. 6a-d and 
a

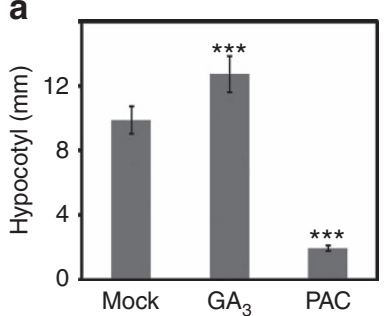

b

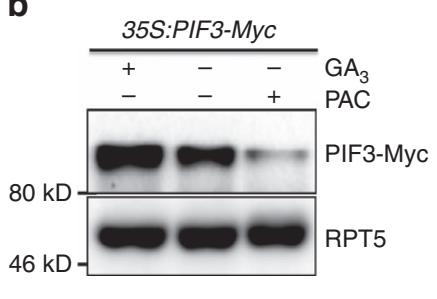

C
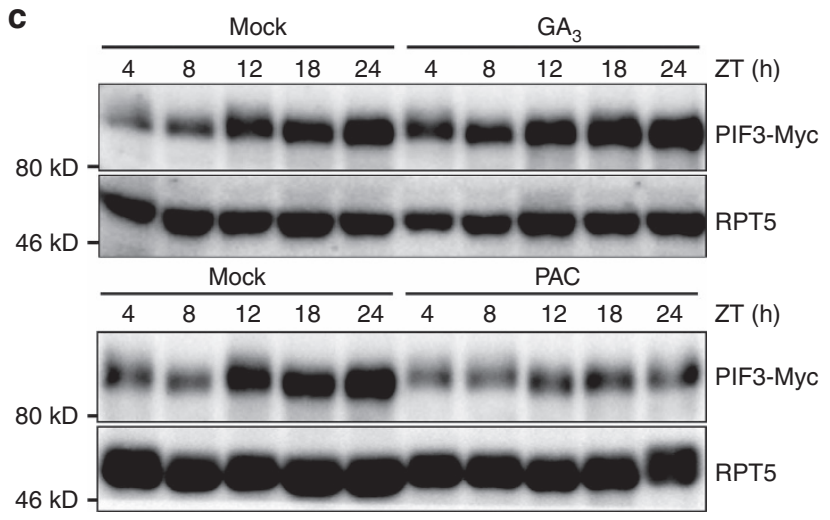

d

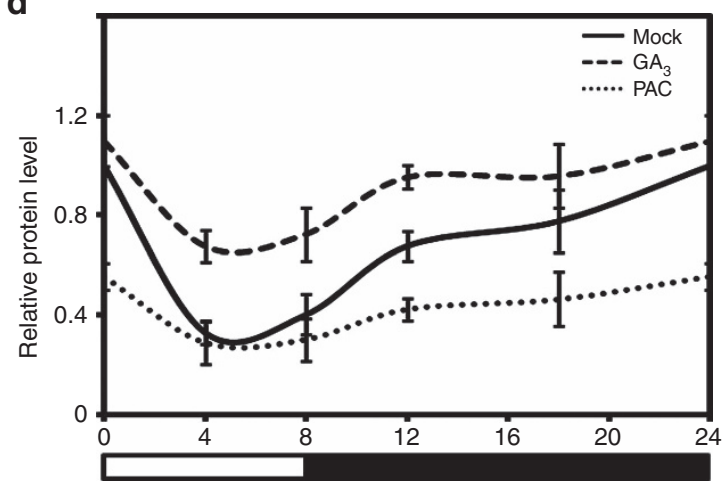

ZT (h)

Figure 4 | DELLAs negatively regulate PIF3 protein abundance under continuous light and diurnal conditions. (a) Hypocotyl lengths of 4-day-old 35S:PIF3-Myc seedlings treated with $\mathrm{GA}_{3}(10 \mu \mathrm{M})$ or PAC $(0.5 \mu \mathrm{M})$ under continuous red light. Means \pm s.d. were calculated from at least 30 seedlings. Statistical significance was determined using the Student's $t$ tests between the $\mathrm{GA}_{3}$ or PAC treatment and Mock. ${ }^{\star \star \star} P<0.001$. (b) PIF3-Myc protein levels of the seedlings shown in (a). Total proteins were analyzed by immunoblots using anti-Myc and anti-RPT5. RPT5 was used as a loading control. (c) Levels of PIF3-Myc proteins in seedlings grown under diurnal conditions. 35S:PIF3-Myc seedlings were grown on the medium without treatment, with $\mathrm{GA}_{3}(10 \mu \mathrm{M})$, or with $\mathrm{PAC}(0.5 \mu \mathrm{M})$ under short-day condition ( $8 \mathrm{~h}$ white light/16 $\mathrm{h}$ darkness) for 5 days, and samples were collected at the indicated times. (d) The quantification of the western blot results from (c) using image J software. PIF3-Myc protein levels were normalized to RPT5 loading control. The value of Mock at ZT24 was set to be 1. Quantitative data are shown as means \pm s.e.m., $n=3$.

Supplementary Fig. 3). The results showed that PAC can induce PIF3 destruction in phyB-9 and lrb123 mutants as effectively as in wild type. These results indicate that phyB and LRBs are not required for DELLA-mediated PIF3 degradation. Conversely, we investigated whether the della pentuple mutant affect lighttriggered degradation of PIF3 protein. As shown in the Fig. 6e,f, the red light-induced degradation rate of PIF3 in della mutant is similar to that in wild type, indicating that DELLAs are not
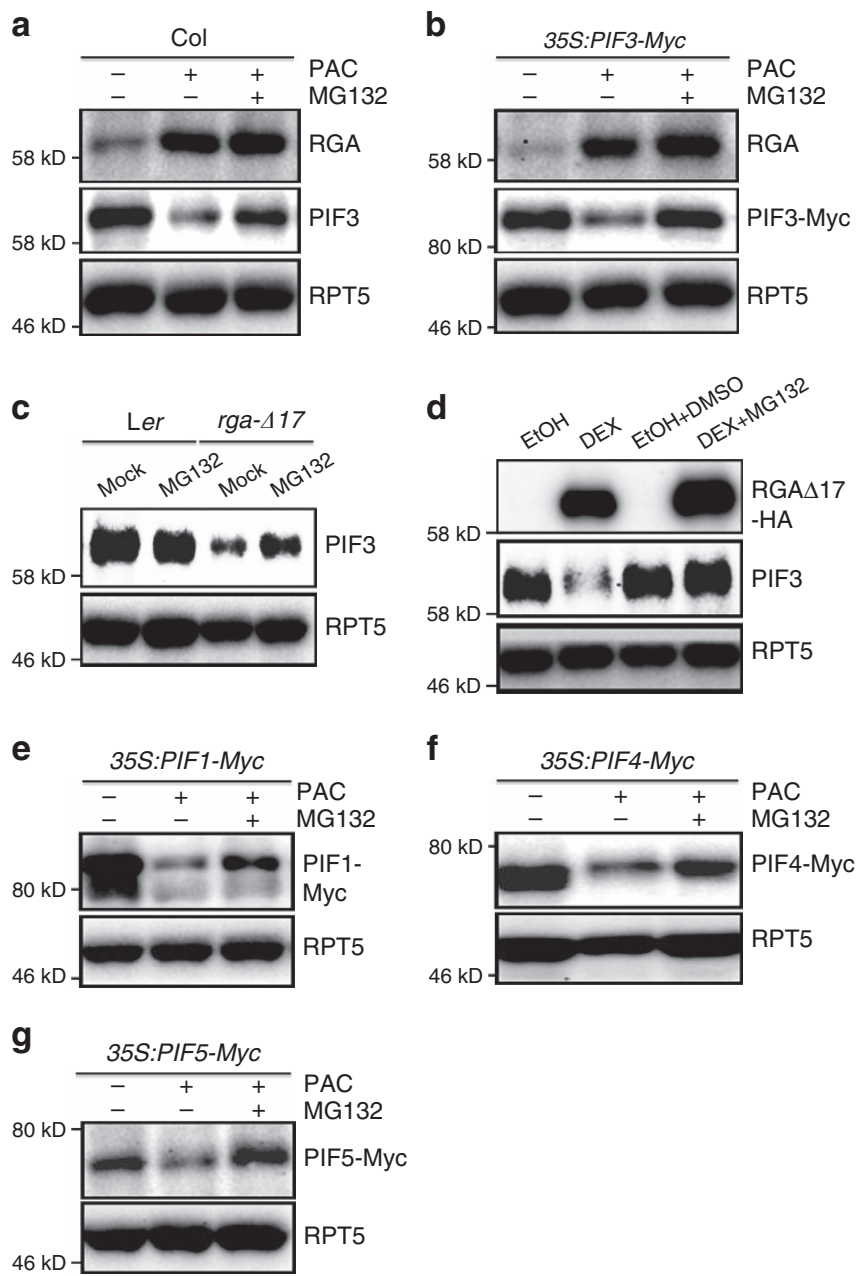

Figure 5 | DELLAs promote the degradation of PIF proteins via the ubiquitin-proteasome system. (a,b) Effects of PAC $(0.5 \mu \mathrm{M})$ and MG132 $(100 \mu \mathrm{M})$ treatments on RGA, PIF3 and PIF3-Myc protein levels in 4-day-old dark-grown seedlings. (c) Effects of MG132 on PIF3 protein levels in 4-day-old dark-grown rga-417 seedlings. (d) Effects of MG132 on PIF3 protein levels after transient induction of RGA $\triangle 17-H A$. Four-day-old darkgrown RGA $\triangle 17-\mathrm{HA}$ seedlings were treated with DEX or DEX plus MG132 for 24h. EtOH and DMSO are the solvents for DEX and MG132, respectively. (e-g) Effects of combinational PAC and MG132 treatments on PIF1-Myc, PIF4-Myc and PIF5-Myc protein levels in 4-day-old dark-grown seedlings. Each immunoblot result is the representative of at least three repeats, and RPT5 was used as a loading control.

required for red light-induced PIF3 degradation via phyB/LRBs. Thus, DELLAs and phyB/LRBs represent two independent pathways that mediate degradation of PIF3.

COP1 and DET1 proteins have been shown to maintain PIF3 protein stability, and PIF3 protein can hardly be detected in cop 1-4 or det1-1 mutant ${ }^{47,48}$. Our analyses showed that GA and PAC could not modulate PIF3 abundance without the presence of COP1 or DET1, indicating that COP1 and DET1 are essential for GA-mediated PIF3 accumulation (Supplementary Fig. 4).

DELLAs regulate PIF3 by both sequestration and degradation. Previous reports indicated that DELLAs interact with PIF3 and prevent PIF3 from binding to its target genes ${ }^{37}$. In this study, we found that DELLAs can promote the degradation of PIF3. To dissect the specific contributions of these two forms of regulation, 
a

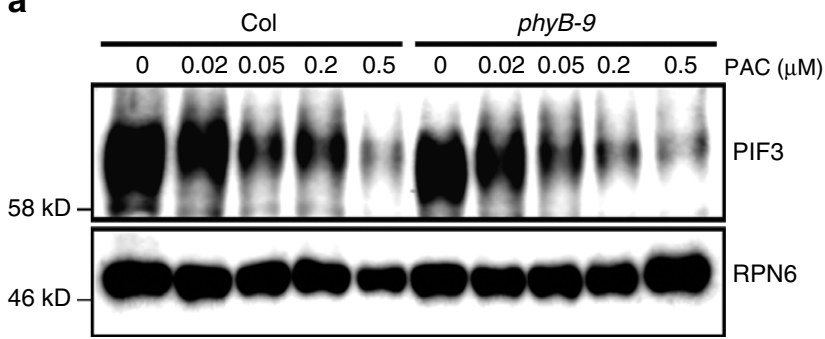

c

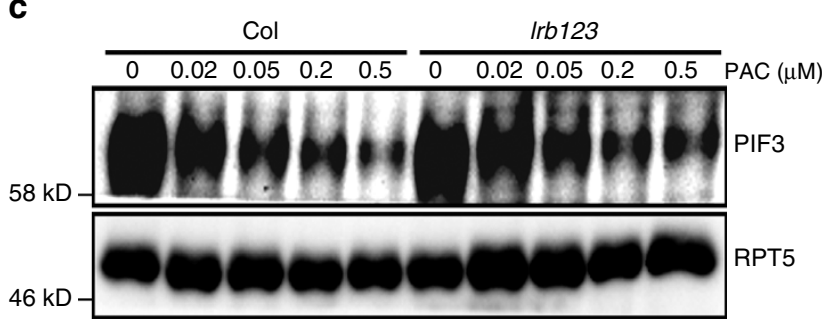

e

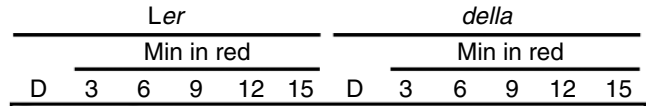

58 kD

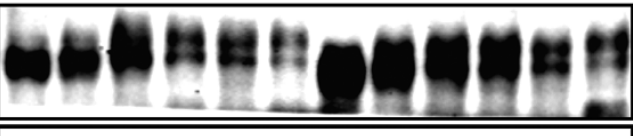

$46 \mathrm{kD}$

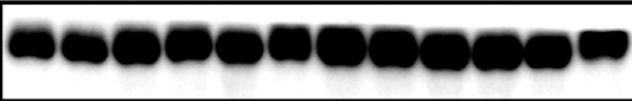

b

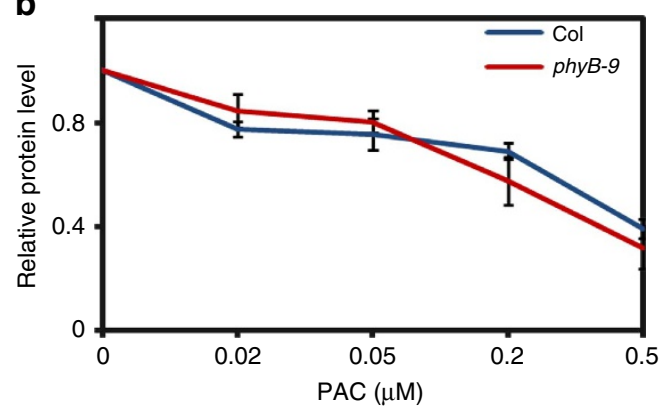

d

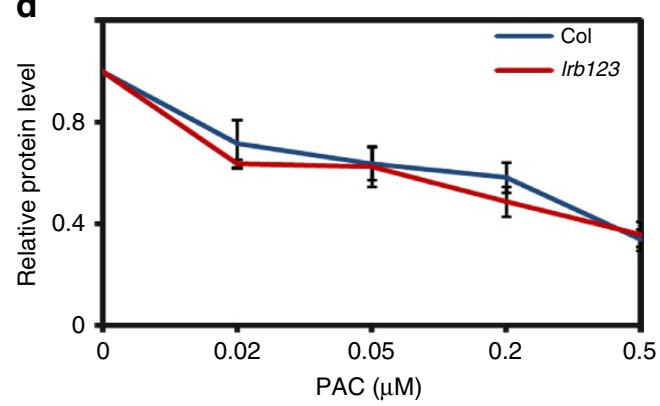

$\mathbf{f}$

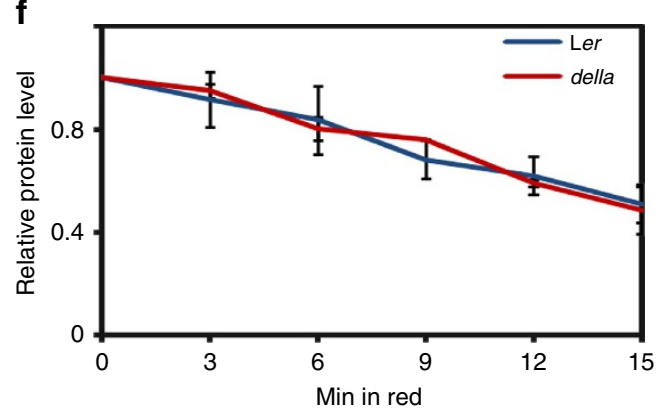

Figure 6 | DELLAs and phyB/LRBs regulate the degradation of PIF3 independently. (a) Endogenous PIF3 protein levels in the 4-day-old dark-grown seedlings of phyB-9 mutant and its wild-type counterparts grown on medium containing different concentrations $(0,0.02,0.05,0.2$ and $0.5 \mu \mathrm{M})$ of PAC. RPN6 was used as a loading control. (b) The quantification of the western blot results from (a) using image J software. PIF3 protein levels were normalized to RPN6 loading control. The value of starting point was set to 1. Quantitative data are shown as means \pm s.e.m., $n=3$. (c) Endogenous PIF3 protein levels in 4-day-old dark-grown Irb1/rb2/rb3 triple-mutant (Irb123) and wild-type seedlings grown on medium containing different concentrations (0, 0.02, 0.05, 0.2 and $0.5 \mu \mathrm{M}$ ) of PAC. RPT5 was used as a loading control. (d) The quantification of the western blot results from (c) using image J software. PIF3 protein levels were normalized to RPT5 loading control. The value of starting point was set to 1. Quantitative data are shown as means \pm s.e.m., $n=3$.

(e) Endogenous PIF3 protein levels in 4-day-old seedlings of della mutant and its wild-type counterparts during dark to red light transition. Dark-grown seedlings were irradiated with red light $\left(5 \mu \mathrm{mol} \mathrm{m} \mathrm{m}^{-2} \mathrm{~s}^{-1}\right)$ for the indicated times. RPT5 was used as loading control. (f) The quantification of the western blot results from (e) using image J software. PIF3 protein levels were normalized to RPT5 loading control. The value of starting point was set to 1.

Quantitative data are shown as means \pm s.e.m., $n=3$.

we examined the promoter occupancy of PIF3-Myc by Chromatin ImmunoPrecipitation (ChIP) experiments, as the readout of PIF3 activity under different PAC and/or MG132 treatments.

Seedlings treated with PAC had increased RGA levels and reduced PIF3-Myc levels (Fig. 5b and Supplementary Fig. 5), presenting a situation in which presumably both sequestration and degradation mechanisms would have a role in regulating PIF3 activity. In contrast, treatment of seedlings with both PAC and MG132 increased RGA abundance, while PIF3-Myc levels were almost the same as mock-treated seedlings, presenting a situation in which only sequestration is likely to have a role. We then analyzed the binding of PIF3 to its known target genes (PIL1, IBH1, ATHB2, ATHB4 and HAT1) ${ }^{13}$. As shown in Fig. 7a, the promoters of these five genes were significantly enriched in the ChIP samples of untreated 35S:PIF3-Myc seedlings (Mock). In
PAC-treated seedlings, the enrichment of these promoters was significantly decreased, indicating that sequestration and degradation together strongly inhibit the binding of PIF3 to its target genes. On the other hand, in seedlings treated with both PAC and MG132, the enrichment levels, although still decreased when compared to mock, were clearly higher than those treated with PAC alone, indicating that sequestration by itself can already inhibit the binding of PIF3 to its targets, while degradation provides additional level of inhibition, presumably reducing PIF3 activity even further. Treatment of MG132 alone without PAC did not affect PIF3-Myc or RGA level (Supplementary Fig. 6a), nor the binding of PIF3-Myc on the targets (Supplementary Fig. 6b).

Although DELLA proteins have no classical DNA-binding domains, they have been shown to directly target some GA-responsive genes such as SCL3 (refs 49,50). To test whether 
a

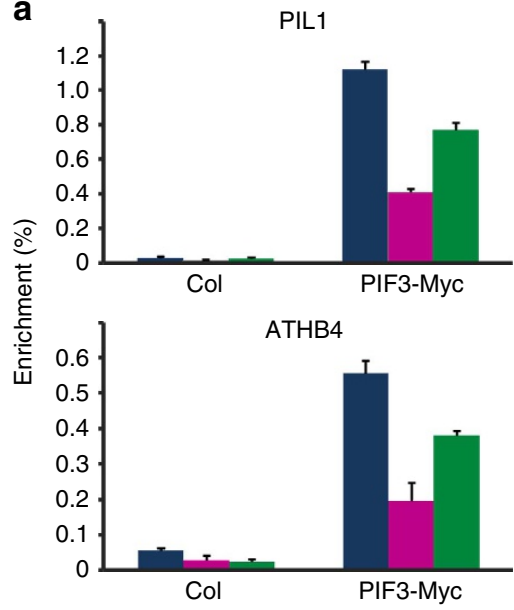

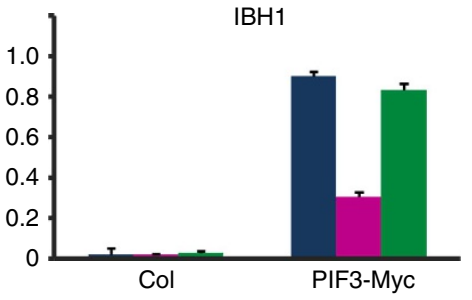
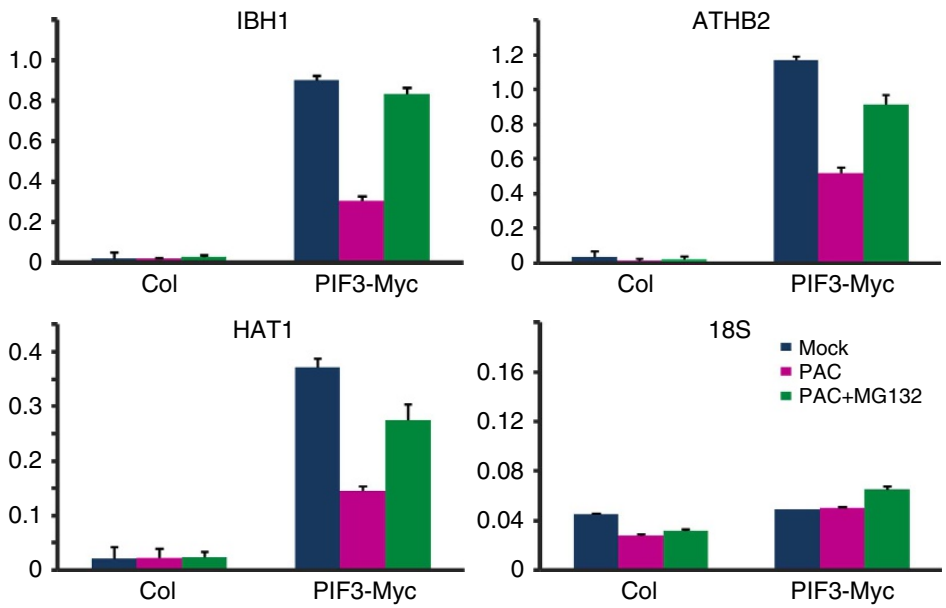

b

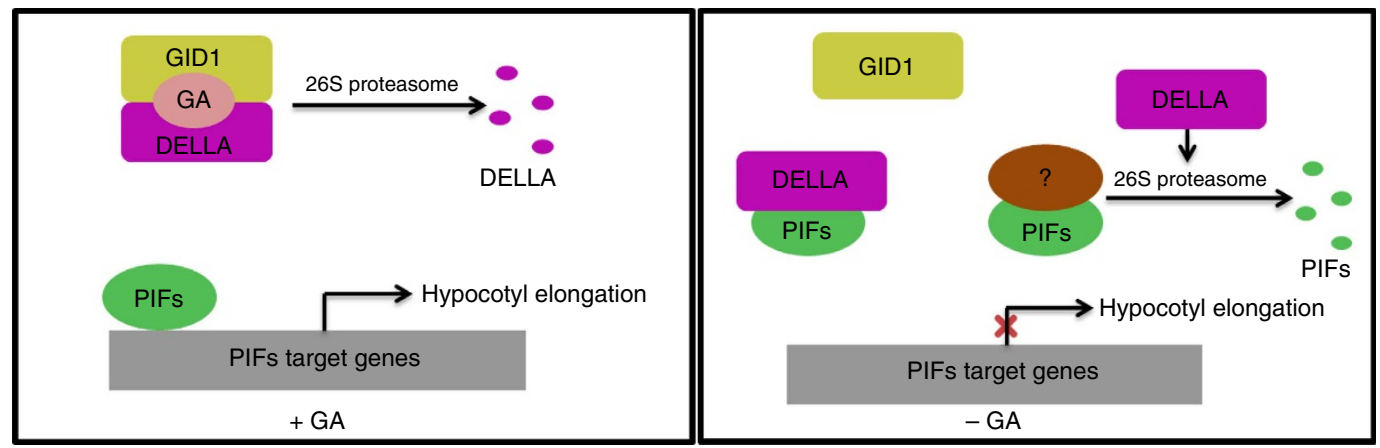

Figure 7 | Both sequestration and degradation of PIF3 by DELLAs reduced the binding activity of PIF3 to its target genes. (a) The ChIP- $q P C R$ analysis of PIF3-Myc binding to its target genes. The 4-day-old wild-type (Col) and 35S:PIF3-Myc seedlings grown in the dark on regular medium, or on medium with $0.5 \mu \mathrm{M}$ PAC and then treated with DMSO or $100 \mu \mathrm{M} \mathrm{MG132}$ for $4 \mathrm{~h}$, were used for ChIP-qPCR analyses. A $18 \mathrm{~S}$ rDNA was used as a non-binding control. The data was calculated from three biological replicates. RGA and PIF3-Myc protein levels in these seedlings are shown in Fig. $5 \mathrm{~b}$ and Supplementary Fig. 5. (b) A model showing that DELLAs inhibit PIFs via both sequestration and degradation to regulate hypocotyl elongation. GA promotes the degradation of DELLAs via 26S proteasome system, and PIFs released from DELLAs can enhance hypocotyl elongation (left panel). When GA is absent, more DELLAs are accumulated, which inhibit the functions of PIFs at two levels: First, DELLAs sequestrate PIFs from binding to their targets 36,37 ; Second, DELLAs promote the degradation of PIFs via $26 \mathrm{~S}$ proteasome. These inhibitions on PIFs together reduce hypocotyl elongation (right panel).

DELLAs potentially interfere in PIF3 binding to its target genes, we conducted a RGA ChIP analysis using the inducible HA-tagged RGA $\Delta 17$ transgenic plants. Our data indicated that DEX-inducible RGA $\triangle 17$-HA protein bound strongly to the SCL3 promoter, but not to the promoters of PIF3 target genes (Supplementary Fig. 7). These results suggest that DELLA proteins would not bind PIF3 target genes.

Taken together, these data suggest that DELLAs can inhibit PIF3 activity via a dual regulatory mechanism, which involves both sequestration and degradation.

\section{Discussion}

Light and GA antagonistically control hypocotyl elongation of seedlings. In this crosstalk, DELLA proteins sequestrate PIF3 and PIF4, and repress their DNA-binding ability, which explains the antagonistic control of hypocotyl elongation by light and $\mathrm{GA}^{36,37}$. In this study, we found that DELLAs negatively regulate PIF3 protein abundance under both continuous dark and light conditions (Figs 1-4). In addition to PIF3 and PIF4, DELLAs also interact with PIF1 and PIF5 (Supplementary Fig. 8 and ref. 38), and promote degradation of these PIFs via the ubiquitinproteasome system (Fig. 5). The reduction of PIF3 abundance by DELLAs contributes significantly to the oscillatory patterns of PIF3 protein level in plants growing in diurnal light/dark conditions (Fig. 4c,d). Furthermore, we showed that both the sequestration and degradation contribute to the inhibition of PIF3 activity (Fig. 7a). Therefore, degradation is a new level of regulation of DELLAs on PIFs, in addition to the previously demonstrated regulation by sequestration. Together, we propose a model that illustrates how GA and light signals coordinate to regulate plant hypocotyl elongation (Fig. $7 \mathrm{~b}$ ). Briefly, we propose that when GA is present, it promotes the formation of the GA-GID1-DELLA complex and leads to the rapid ubiquitination and degradation of DELLAs, thus releasing PIFs to promote hypocotyl elongation. In contrast, when GA is absent, we propose that DELLA proteins accumulate and inhibit the function of PIFs by both sequestrating them from binding to their target promoters as well as by promoting their degradation via the ubiquitin-proteasome system, and thus hypocotyl elongation is inhibited. DELLA-induced PIF3 degradation is independent of the light-mediated PIF3 degradation pathway, as it can occur in the absence of activated phyB and LRBs E3 ligase system. As to how DELLAs mediate the PIF3 abundance, COP1 and DET1 proteins were shown to be essential for GA-mediated PIF3 accumulation (Supplementary Fig. 4), but the E3 ligase through which DELLAs trigger PIF3 degradation is still unknown. GA $_{3}$ application led to a quick reduction of RGA protein levels, but PIF3 abundance was almost fully recovered after $12 \mathrm{~h}$ treatment (Fig. 3i). During this period, the unknown E3 ligase responsible 
for DELLAs-mediated PIF3 degradation may be de-activated, and then PIF3 proteins may accumulate gradually.

GAs can also coordinate with other signals to regulate plant development. For example, core transcription factors in several different signalling pathways, including BZR1, EIN3, JAZ and WD-Repeat/bHLH/MYB complex, were shown to be sequestrated and inhibited by DELLAs, similar to PIF3 and PIF4 (refs 36-44). These results suggest that sequestration is a general mechanism by which DELLAs inhibit transcription factors. Among these transcription factors, BZR1 was the only one for which DELLA was reported to promote a reduction in protein levels ${ }^{41}$. Here, we showed that DELLAs can promote the degradation of PIF1, PIF3, PIF4 and PIF5. Extending from this finding, we propose that controlling protein abundance of transcription factors represents another general mechanism of how DELLAs may regulate their targets. To test this hypothesis, we examined EIN3 from the ethylene signalling pathway. A previous study showed that DELLAs sequester EIN3, but $\mathrm{GA}_{3}$ did not markedly affect EIN3 protein abundance ${ }^{42}$. Consistently, GA treatments did not noticeably affect the abundance of EIN3-GFP and EIN3-FLAG proteins in our experiment; however, PAC treatments dramatically reduced their abundance (Supplementary Fig. 9a,b). It is possible that EIN3 accumulates to a high level in darkness, and is not sensitive to exogenous GA, which decreases DELLA levels but could not further increase EIN3 abundance. On the other hand, highly elevated levels of DELLAs after PAC treatment may promote EIN3 degradation. Taken together, our data suggests that the inhibition of transcription factors by DELLAs is mediated through both sequestration and degradation.

\section{Methods}

Plant materials and growth conditions. The wild-type Arabidopsis ecotypes used in this study were Columbia-0 (Col-0), Landsberg erecta (Ler) and Wassilewskija (WS). The mutants and transgenic lines were described previously: pif3-3 (ref. 4), 35S: PIF1-Myc, 35S: PIF3-Myc, 35S: PIF4-Myc and 35S: PIF5-Myc (ref. 48), rga-24 (ref. 25), rga-417 (ref. 26), della (gai-t6 rga-t2 rgl1-1 rgl2-1 rgl3-1) (ref. 37), sly1-10 gai-t6, sly1-10 rga-24, sly1-10 gai-t6 rga-24 (ref. 34), phyB-9 (ref. 51), lrb123 (ref. 16), cop1-4 (ref. 52), det1-1(ref. 53), 35S: EIN3-GFP/ein3 eil1 (ref. 54) and 35S: EIN3-FLAG/ein3 eill (ref. 55). Since homozygous $\mathrm{rga}-417$ is sterile ${ }^{26}$, the homozygous $\mathrm{rga}-\Delta 17$ seedlings segregated from the progenies of heterozygous rga- $\Delta 17$ were used for experiments. gai (Col background) is a gift from Xiangdong $\mathrm{Fu}$ of IGDB. All seeds were surface-sterilized and sown on Murashige and Skoog (MS) medium containing $1 \%$ sugar. Seeds were cold-treated at $4{ }^{\circ} \mathrm{C}$ for 4 days in the dark before germination. The seedlings were grown in the dark, under continuous red light $\left(0.5 \mu \mathrm{mol} \mathrm{m}^{-2} \mathrm{~s}^{-1}\right)$ or under short-day conditions ( $8 \mathrm{~h}$ white light $\left(85 \mu \mathrm{mol} \mathrm{m} \mathrm{m}^{-2} \mathrm{~s}^{-1}\right)+16 \mathrm{~h}$ dark) for the indicated times, unless indicated otherwise. Manipulation of seedlings in darkness was performed under dim green light.

Generation of transgenic plants. DNA fragments containing two deletion mutants (RGAL17 and GAIA17) without stop codons were amplified and inserted into the XmaI and PstI restriction sites of a pBSK-derived plasmid containing triple HA-tag to make constructs pBSK-RGA $\Delta 17-\mathrm{HA}$ and pBSK-GAI $\Delta 17-\mathrm{HA}$. Further, the fragments RGA $\triangle 17-H A$ and $G A I \Delta 17-H A$ were amplified from pBSK-RGA $\Delta 17$ HA or pBSK-GAI $\Delta 17-H A$, and these fragments were digested with the SalI and SpeI, and then ligated into the XhoI and SpeI digested binary vector pTA7002 (ref. 56) to generate pTA7002-RGA $\Delta 17$-HA and pTA7002-GAI $\Delta 17-\mathrm{HA}$ constructs. These binary constructs were introduced into the GV3101 strain of Agrobacterium and transformed into della mutant plants, using the floral dip transformation method ${ }^{57}$. The transformants were selected on MS medium containing $50 \mathrm{mg} \mathrm{ml}^{-1}$ hygromycin B (Sigma-Aldrich), and named as RGA $\Delta 17-\mathrm{HA}$ and GAI $\Delta 17-\mathrm{HA}$, respectively. The primers are listed in Supplementary Table 2.

Plant treatments. $\mathrm{GA}_{3}(\mathrm{GA})$, paclobutrazol (PAC) and DEX were dissolved in ethanol. The proteasome inhibitor MG132 was dissolved in DMSO. For continuous $\mathrm{GA}_{3}$ and PAC treatments, after the seeds were induced by white light for germination on regular MS medium, they were transferred to medium containing $\mathrm{GA}_{3}$ $(10 \mu \mathrm{M}), \mathrm{PAC}(0.5 \mu \mathrm{M})$ and ethanol $(0.01 \%(\mathrm{v} / \mathrm{v})$, as control), and then grown in the dark for 4 days. For the transient treatment by $\mathrm{GA}_{3}$, the seedlings were collected and vacuum-infiltrated with liquid MS medium containing $100 \mu \mathrm{M} \mathrm{GA}_{3}$ or ethanol alone (as control) for $10 \mathrm{~min}$ and then kept immersed in the same solution for the indicated times. For continuous DEX treatment, Arabidopsis seedlings were grown on the MS medium containing $1 \mu \mathrm{M}$ DEX or ethanol alone (as control). For the transient induction by DEX, the 4-day-old seedlings were vacuum-infiltrated with liquid MS medium containing $10 \mu \mathrm{M}$ DEX or ethanol alone (as control) for $10 \mathrm{~min}$ and then kept immersed in the same solution for the indicated times. For MG132 treatment, the 4-day-old seedlings were vacuum-infiltrated with liquid MS medium containing $100 \mu \mathrm{M}$ MG132 (dissolved in DMSO) or DMSO alone (as control) for $10 \mathrm{~min}$ and kept immersed in the same solution for $4 \mathrm{~h}$ unless indicated otherwise.

Protein extraction and Immunoblots. Total proteins were extracted by homogenizing seedlings using denaturing buffer $\left(100 \mathrm{mM} \mathrm{NaH}_{2} \mathrm{PO} 4,10 \mathrm{mM}\right.$ Tris- $\mathrm{HCl}$ $\mathrm{pH}$ 8.0, $8 \mathrm{M}$ urea, $1 \mathrm{mM}$ phenylmethylsulfonyl fluoride, and $\times 1$ complete protease inhibitor mixture (Roche). Seedlings were growing in the dark for 4 days unless specific indications. Extracts were centrifuged at $16,000 \mathrm{~g}$ for $10 \mathrm{~min}$ at $4{ }^{\circ} \mathrm{C}$, and protein concentration in the supernatants was quantified by the Bradford assay. Aliquots of denatured total protein were separated on $8 \%$ SDS-PAGE gels and transferred to PVDF membranes. Anti-PIF3 purified antibody at 1:500 (v/v) dilution (ref. 48), anti-Myc polyclonal antibody (Sigma-Aldrich, Cat. No: C3956) at 1:1,000 (v/v) dilution, anti-HA antibody (Sigma-Aldrich, Cat. No: H9658) at 1:1,000 (v/v) dilution, anti-RGA antibody (Agrisera, Cat. No: AS111630) at 1:1,000 (v/v) dilution, anti-FLAG (Sigma-Aldrich, Cat. No: F3165) at 1:1,000 (v/v) dilution, anti-GFP (Abmart, Cat. No: M20004) at 1:1,000 (v/v) dilution, anti-RPN6 polyclonal antibody (ref. 58) at 1:2,000 (v/v) dilution, and anti-PRT5 polyclonal antibody (ref. 58) at 1:2,000 (v/v) dilution were used as primary antibodies. Each experiment was repeated at least three times, and one representative result was shown. Quantification results of immunoblots in Figs 3f, 4d, 6b, 6d and 6f were quantified by Image J software. Supplementary Figures 10-22 are original full versions of the immunoblot images.

RNA extraction and quantitative RT-PCR. Total RNA was extracted using the RNeasy plant mini kit (Qiagen). cDNA was synthesized by ReverTra Ace qPCR RT Master Mix (TOYOBO). The quantitative RT-PCR analysis was performed using SYBR Premix Ex Taq (Takara) in an ABI 7500 fast real-time instrument. Each experiment was repeated with three biological samples, and RT-PCR reactions were performed with three technical replicates for each sample. The primers are listed in Supplementary Table 2.

Hypocotyl lengths measurement. After the indicated times of growth and treatment, at least 30 seedlings were laid on the agar plates, and digital pictures were taken. Then, the hypocotyl lengths were measured using Image J software.

In vitro pull down assays. The constructs for expressing His-PIF1, His-PIF3, GST-PIF4 and GST-PIF5 were described previously ${ }^{48}$. Full-length RGA fragment was inserted into pMal-C2X vector to fuse with maltose-binding protein (MBP). All constructs were expressed in E. coli strain BL21 under the induction of $1 \mathrm{mM}$ IPTG (isopropyl- $\beta$-D-thiogalactopyranoside). Two micrograms MBP or MBP-RGA proteins were mixed with $2 \mu \mathrm{g}$ His- or GST-tagged proteins in $500 \mu \mathrm{l}$ binding buffer ( $20 \mathrm{mM}$ Tris-HCl, $\mathrm{pH} 7.5,150 \mathrm{mM} \mathrm{NaCl}$ and $0.1 \%$ Nonidet P-40), and the mixture was rotated at $4{ }^{\circ} \mathrm{C}$ for $2 \mathrm{~h}$. The amylase agarose beads were washed with binding buffer for three times and then added into the mixture. Then, the mixture was rotated at $4^{\circ} \mathrm{C}$ for another $2 \mathrm{~h}$. After being washed for five times with binding buffer, the MBP resin was boiled with protein loading buffer and analyzed by immunoblots. Anti-His (Sigma-Aldrich, Cat. No: H1029-.2ML) at 1:1,000 dilution, anti-GST (Sigma-Aldrich, Cat. No: G1160-.2ML) at 1:5,000 dilution and anti-MBP (New England Biolabs, Cat. No: E8032S) at 1:5,000 dilution were used for the western blots.

Bimolecular fluorescence complementation assay. The full-length cDNA of RGA was amplified and inserted into the SpeI and BamHI sites of pSY736 $\left(\mathrm{YFP}^{\mathrm{N}}\right)$ vector, resulting in plasmid YFP $^{\mathrm{N}}$-RGA. YFPC-PIF1, YFPC - PIF3, YFPC-PIF4 and YFP $^{\mathrm{C}}$-PIF5 plasmids were described previously ${ }^{48}$. The plasmids were extracted and concentrated to $2 \mathrm{mg} \mathrm{ml}^{-1}$. The particle-mediated transformation using onion epidermal cells was performed ${ }^{59}$. After $24 \mathrm{~h}$ of incubation, YFP signal was detected using a Zeiss LSM 710 confocal microscope. The primers used for plasmid construction were listed in Supplementary Table 2.

\section{Chromatin immunoprecipitations assay. ChIP assays were performed as} described previously ${ }^{37}$. 35S:PIF3-Myc seedlings were grown for 4 days on the MS medium containing $0.5 \mu \mathrm{M}$ PAC or EtOH in darkness, and were collected and treated with DMSO or $100 \mu \mathrm{M}$ MG132 for $4 \mathrm{~h}$. RGA $\Delta 17$-HA seedlings were grown for 4 days in the dark, and were collected and infiltrated with or without $10 \mu \mathrm{M}$ DEX for $24 \mathrm{~h}$. The samples $(2 \mathrm{~g})$ were treated with $15 \mathrm{ml}$ of $1 \%$ formaldehyde under vacuum infiltration for $15 \mathrm{~min}$, and then $1 \mathrm{ml} 2 \mathrm{M}$ glycine was added to stop crosslinking for $5 \mathrm{~min}$. For the ChIP analysis used 35S: PIF3-Myc seedlings, the solubilized chromatin was immunoprecipitated by $30 \mu \mathrm{l}$ EZview Red Anti-c-Myc Affinity Gel (Sigma-Aldrich, Cat. No: E6654) at $4{ }^{\circ} \mathrm{C}$ for $5 \mathrm{~h}$. For the ChIP analysis using RGA $\Delta 17-\mathrm{HA}$ seedlings, the solubilized chromatin was mixed with $10 \mu \mathrm{l}$ 
anti-HA antibody (Sigma) and incubated at $4{ }^{\circ} \mathrm{C}$ for $1 \mathrm{~h}$. Then, $40 \mu \mathrm{l}$ Dynabeads Protein G (Life Technologies, Cat. No: 10003D) was added, and the sample was incubated overnight at $4{ }^{\circ} \mathrm{C}$. The coimmunoprecipitated DNA was recovered and analyzed by quantitative PCR. All primers used in ChIP assays were listed in Supplementary Table 2 .

Data availability. The authors declare that all data supporting the findings of this study are available within the article and its Supplementary Information files or are available upon request from the corresponding authors.

\section{References}

1. Von Arnim, A. \& Deng, X. W. Light control of seedling development. Ann. Rev. Plant. Physiol. Mol. Biol. 47, 215-243 (1996).

2. Ni, M., Tepperman, J. M. \& Quail, P. H. PIF3, a phytochrome-interacting factor necessary for normal photoinduced signal transduction, is a novel basic helixloop-helix protein. Cell 95, 657-667 (1998).

3. Kim, J. Y. et al. Functional characterization of phytochrome interacting factor 3 in phytochrorne-mediated light signal transduction. Plant Cell 15, 2399-2407 (2003).

4. Monte, E. et al. The phytochrome-interacting transcription factor, PIF3, acts early, selectively, and positively in light-induced chloroplast development. Proc. Natl Acad. Sci. USA 101, 16091-16098 (2004).

5. Huq, E. \& Quail, P. H. PIF4, a phytochrome-interacting bHLH factor, functions as a negative regulator of phytochrome B signalling in Arabidopsis. EMBO J. 21, 2441-2450 (2002)

6. Huq, E. et al. PHYTOCHROME-INTERACTING FACTOR 1 is a critical bHLH regulator of chlorophyll biosynthesis. Science 305, 1937-1941 (2004).

7. Khanna, R. et al. A novel molecular recognition motif necessary for targeting photoactivated phytochrome signalling to specific basic helix-loop-helix transcription factors. Plant Cell 16, 3033-3044 (2004).

8. Leivar, P. et al. Multiple phytochrome-interacting bHLH transcription factors repress premature seedling photomorphogenesis in darkness. Curr. Biol. 18, 1815-1823 (2008).

9. Shin, J. et al. Phytochromes promote seedling light responses by inhibiting four negatively-acting phytochrome-interacting factors. Proc. Natl Acad. Sci. USA 106, 7660-7665 (2009).

10. Leivar, P. et al. Dynamic antagonism between phytochromes and PIF family basic helix-loop-helix factors induces selective reciprocal responses to light and shade in a rapidly responsive transcriptional network in Arabidopsis. Plant Cell 24, 1398-1419 (2012).

11. Leivar, P. et al. Definition of early transcriptional circuitry involved in light-induced reversal of PIF-imposed repression of photomorphogenesis in young Arabidopsis seedlings. Plant Cell 21, 3535-3553 (2009).

12. Leivar, P. \& Quail, P. H. PIFs: pivotal components in a cellular signalling hub. Trends Plant Sci. 16, 19-28 (2011).

13. Zhang, Y. et al. A quartet of PIF bHLH factors provides a transcriptionally centered signalling hub that regulates seedling morphogenesis through differential expression-patterning of shared target genes in Arabidopsis. PLoS Genet. 9, e1003244 (2013).

14. Al-Sady, B., Ni, W., Kircher, S., Schafer, E. \& Quail, P. H. Photoactivated phytochrome induces rapid PIF3 phosphorylation prior to proteasomemediated degradation. Mol. Cell 23, 439-446 (2006).

15. Shen, Y., Khanna, R., Carle, C. M. \& Quail, P. H. Phytochrome induces rapid PIF5 phosphorylation and degradation in response to red-light activation. Plant Physiol. 145, 1043-1051 (2007).

16. Ni, W. M. et al. A mutually assured destruction mechanism attenuates light signalling in Arabidopsis. Science 344, 1160-1164 (2014).

17. Shen, H. et al. Light-induced phosphorylation and degradation of the negative regulator phytochrome-interacting factor 1 from Arabidopsis depend upon its direct physical interactions with photoactivated phytochromes. Plant Cell 20, 1586-1602 (2008)

18. Castillon, A., Shen, H. \& Huq, E. Phytochrome Interacting Factors: central players in phytochrome-mediated light signalling networks. Trends Plant Sci. 12, 514-521 (2007)

19. Jiao, Y. L., Lau, O. S. \& Deng, X. W. Light-regulated transcriptional networks in higher plants. Nat. Rev. Genet. 8, 217-230 (2007).

20. Bae, G. \& Choi, G. Decoding of light signals by plant phytochromes and their interacting proteins. Annu. Rev. Plant Biol. 59, 281-311 (2008).

21. Alabadi, D., Gil, J., Blazquez, M. A. \& Garcia-Martinez, J. L. Gibberellins repress photomorphogenesis in darkness. Plant Physiol. 134, 1050-1057 (2004).

22. Sun, T. P. Gibberellin metabolism, perception and signalling pathways in Arabidopsis. Arabidopsis book 6, e0103 (2008)

23. Koornneef, M. et al. A gibberellin insensitive mutant of Arabidopsis thaliana. Physiol. Plantarum 65, 33-39 (1985).

24. Peng, J. R. et al. The Arabidopsis GAI gene defines a signalling pathway that negatively regulates gibberellin responses. Gene Dev. 11, 3194-3205 (1997)
25. Silverstone, A. L., Mak, P. Y. A., Martinez, E. C. \& Sun, T. P. The new RGA locus encodes a negative regulator of gibberellin response in Arabidopsis thaliana. Genetics 146, 1087-1099 (1997).

26. Dill, A., Jung, H. S. \& Sun, T. P. The DELLA motif is essential for gibberellininduced degradation of RGA. Proc. Natl Acad. Sci. USA 98, 14162-14167 (2001).

27. Silverstone, A. L. et al. Repressing a repressor: gibberellin-induced rapid reduction of the RGA protein in Arabidopsis. Plant Cell 13, 1555-1565 (2001)

28. Wen, C. K. \& Chang, C. Arabidopsis RGL1 encodes a negative regulator of gibberellin responses. Plant Cell 14, 87-100 (2002).

29. Lee, S. C. et al. Gibberellin regulates Arabidopsis seed germination via RGL2, a GAI/RGA-like gene whose expression is up-regulated following imbibition. Gene Dev. 16, 646-658 (2002).

30. Tyler, L. et al. DELLA proteins and gibberellin-regulated seed germination and floral development in Arabidopsis. Plant Physiol. 135, 1008-1019 (2004).

31. Reed, J. W., Foster, K. R., Morgan, P. W. \& Chory, J. Phytochrome B affects responsiveness to gibberellins in Arabidopsis. Plant Physiol. 112, 337-342 (1996).

32. Achard, P. et al. DELLAs contribute to plant photomorphogenesis. Plant Physiol. 143, 1163-1172 (2007).

33. McGinnis, K. M. et al. The Arabidopsis SLEEPY1 gene encodes a putative F-box subunit of an SCF E3 ubiquitin ligase. Plant Cell 15, 1120-1130 (2003).

34. Dill, A., Thomas, S. G., Hu, J., Steber, C. M. \& Sun, T. P. The Arabidopsis F-box protein SLEEPY1 targets gibberellin signalling repressors for gibberellininduced degradation. Plant Cell 16, 1392-1405 (2004).

35. Fu, X. et al. The Arabidopsis mutant sleepylgar2-1 protein promotes plant growth by increasing the affinity of the SCFSLY1 E3 ubiquitin ligase for DELLA protein substrates. Plant Cell 16, 1406-1418 (2004).

36. de Lucas, M. et al. A molecular framework for light and gibberellin control of cell elongation. Nature 451, 480-484 (2008).

37. Feng, S. et al. Coordinated regulation of Arabidopsis thaliana development by light and gibberellins. Nature 451, 475-479 (2008).

38. Gallego-Bartolome, J. et al. Transcriptional diversification and functional conservation between DELLA proteins in Arabidopsis. Mol. Biol. Evol. 27, 1247-1256 (2010).

39. Bai, S. et al. PIF3 is involved in the primary root growth inhibition of Arabidopsis induced by nitric oxide in the light. Mol. Plant 7, 616-625 (2014).

40. Bai, M. Y. et al. Brassinosteroid, gibberellin and phytochrome impinge on a common transcription module in Arabidopsis. Nat. Cell Biol. 14, 810-U878 (2012).

41. Li, Q. F. et al. An interaction between BZR1 and DELLAs mediates direct signalling crosstalk between brassinosteroids and gibberellins in Arabidopsis. Sci. Signal 5, ra72 (2012).

42. An, F. et al. Coordinated regulation of apical hook development by gibberellins and ethylene in etiolatedArabidopsis seedlings. Cell. Res. 22, 915-927 (2012).

43. Hou, X. L., Lee, L. Y. C., Xia, K. F., Yen, Y. Y. \& Yu, H. DELLAs modulate jasmonate signalling via competitive binding to JAZs. Dev. Cell. 19, 884-894 (2010).

44. Qi, T. et al. Arabidopsis DELLA and JAZ proteins bind the WD-Repeat/bHLH/ MYB complex to modulate gibberellin and jasmonate signalling synergy. Plant Cell 26, 1118-1133 (2014).

45. Arana, M. V., Marin-de la Rosa, N., Maloof, J. N., Blazquez, M. A. \& Alabadi, D. Circadian oscillation of gibberellin signalling in Arabidopsis. Proc. Natl Acad. Sci. USA 108, 9292-9297 (2011).

46. Soy, J. et al. Phytochrome-imposed oscillations in PIF3 protein abundance regulate hypocotyl growth under diurnal light/dark conditions in Arabidopsis. Plant J. 71, 390-401 (2012).

47. Bauer, D. et al. Constitutive photomorphogenesis 1 and multiple photoreceptors control degradation of phytochrome interacting factor 3 , a transcription factor required for light signalling in Arabidopsis. Plant Cell 16, 1433-1445 (2004).

48. Dong, J. et al. Arabidopsis DE-ETIOLATED1 represses photomorphogenesis by positively regulating phytochrome-interacting factors in the dark. Plant Cell 26, 3630-3645 (2014).

49. Zentella, R. et al. Global analysis of della direct targets in early gibberellin signalling in Arabidopsis. Plant Cell 19, 3037-3057 (2007).

50. Park, J., Nguyen, K. T., Park, E., Jeon, J. S. \& Choi, G. DELLA proteins and their interacting RING finger proteins repress gibberellin responses by binding to the promoters of a subset of gibberellin-responsive genes in Arabidopsis. Plant Cell 25, 927-943 (2013).

51. Reed, J. W., Nagpal, P., Poole, D. S., Furuya, M. \& Chory, J. Mutations in the gene for the red/far-red light receptor phytochrome B alter cell elongation and physiological responses throughout Arabidopsis development. Plant Cell 5, 147-157 (1993). 
52. McNellis, T. W. et al. Genetic and molecular analysis of an allelic series of cop1 mutants suggests functional roles for the multiple protein domains. Plant Cell 6, 487-500 (1994).

53. Chory, J., Peto, C., Feinbaum, R., Pratt, L. \& Ausubel, F. Arabidopsis thaliana mutant that develops as a light-grown plant in the absence of light. Cell 58, 991-999 (1989).

54. He, W. et al. A small-molecule screen identifies L-kynurenine as a competitive inhibitor of TAA1/TAR activity in ethylene-directed auxin biosynthesis and root growth in Arabidopsis. Plant Cell 23, 3944-3960 (2011).

55. An, F. et al. Coordinated regulation of apical hook development by gibberellins and ethylene in etiolated Arabidopsis seedlings. Cell. Res. 22, 915-927 (2012).

56. Aoyama, T. \& Chua, N. H. A glucocorticoid-mediated transcriptional induction system in transgenic plants. Plant J. 11, 605-612 (1997).

57. Clough, S. J. \& Bent, A. F. Floral dip: a simplified method for Agrobacteriummediated transformation of Arabidopsis thaliana. Plant J. 16, 735-743 (1998).

58. Peng, Z. et al. The cellular level of PR500, a protein complex related to the $19 \mathrm{~S}$ regulatory particle of the proteasome, is regulated in response to stresses in plants. Mol. Biol. Cell 12, 383-392 (2001).

59. von Arnim, A. Subcellular localization of GUS- and GFP-tagged proteins in onion epidermal cells. CSH Protoc. 2007, pdb.prot4689 (2007).

\section{Acknowledgements}

We thank P.H. Quail for lrb123, 35S:PIF1-Myc, 35S:PIF3-Myc, 35S:PIF4-Myc, and 35S:PIF5-Myc seeds; H. Guo for 35S:EIN3-GFP/ein3 eil1 and 35S:EIN3-Flag/ein3 eil1 seeds; T.P. Sun for sly1-10, sly1-10 gai-t6, sly1-10 rga-24, and sly1-10 gai-t6 rga-24 seeds; $\mathrm{X}$. Fu for gai seed; G. Gusmaroli for assistance in generation of RGA $\Delta 17-\mathrm{HA}$ and GAI $\Delta 17$-HA transgenic plants; Y. Wang for preliminary experiments. We also thank S. Feng of UCLA for critical reading and comments on the manuscript. This work was supported by grants from the National Natural Science Foundation of China (31330048 to X.W.D. and 31271294 to H.C.), National Program on Key Basic Research Project of
China (2012CB910900 to X.W.D.), NIH of United States (GM-47850 to N.W.), PekingTsinghua Center for Life Sciences, and State Key Laboratory of Protein and Plant Gene Research.

\section{Author contributions}

H.C. and X.W.D. conceived and supervised the research. K.L., L.-M.F., H.C. and X.W.D designed the experiments. K.L. performed most of the experiments. R.Y. generated and purified the PIF3 antibody. K.L., N.W., H.C. and X.W.D. wrote the manuscript. All authors discussed the results and made contributions to the manuscript.

\section{Additional information}

Supplementary Information accompanies this paper at http://www.nature.com/ naturecommunications

Competing financial interests: The authors declare no competing financial interests.

Reprints and permission information is available online at http://npg.nature.com/ reprintsandpermissions/

How to cite this article: Li, K. et al. DELLA-mediated PIF degradation contributes to coordination of light and gibberellin signalling in Arabidopsis. Nat. Commun. 7:11868 doi: $10.1038 /$ ncomms11868 (2016).

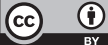

This work is licensed under a Creative Commons Attribution 4.0 International License. The images or other third party material in this article are included in the article's Creative Commons license, unless indicated otherwise in the credit line; if the material is not included under the Creative Commons license, users will need to obtain permission from the license holder to reproduce the material. To view a copy of this license, visit http://creativecommons.org/licenses/by/4.0/ 